\title{
Modeling the effects of drug resistant influenza virus in a pandemic Stefan O Brockmann ${ }^{1}$, Markus Schwehm², Hans-Peter Duerr ${ }^{2}$, Mark Witschi ${ }^{3}$, Daniel Koch ${ }^{3}$, Beatriz Vidondo ${ }^{3}$ and Martin Eichner*2
}

\begin{abstract}
Address: ${ }^{1}$ Department of Epidemiology and Health Reporting, Baden-Württemberg State Health Office, District Government Stuttgart, Germany, ${ }^{2}$ Department of Medical Biometry, University of Tübingen, Germany and ${ }^{3}$ Swiss Federal Office for Public Health, Bern, Switzerland

Email: Stefan O Brockmann - stefan.brockmann@rps.bwl.de; Markus Schwehm - markus.schwehm@explosys.de; Hans-Peter Duerr - hanspeter.duerr@uni-tuebingen.de; Mark Witschi - mark.witschi@gmail.com; Daniel Koch - Daniel.koch@bag.admin.ch; Beatriz Vidondo - beatriz.vidondo@bag.admin.ch; Martin Eichner* - martin.eichner@uni-tuebingen.de

* Corresponding author
\end{abstract}

Published: 30 October 2008

Virology Journal 2008, 5:133 doi:10.1186/1743-422X-5-133

This article is available from: http://www.virologyj.com/content/5/I/I33

This is an Open Access article distributed under the terms of the Creative Commons Attribution License (http://creativecommons.org/licenses/by/2.0), which permits unrestricted use, distribution, and reproduction in any medium, provided the original work is properly cited.

\begin{abstract}
Neuraminidase inhibitors $(\mathrm{NI})$ play a major role in plans to mitigate future influenza pandemics. Modeling studies suggested that a pandemic may be contained at the source by early treatment and prophylaxis with antiviral drugs. Here, we examine the influence of NI resistant influenza strains on an influenza pandemic. We extend the freely available deterministic simulation program InfluSim to incorporate importations of resistant infections and the emergence of de novo resistance. The epidemic with the fully drug sensitive strain leads to a cumulative number of 19,500 outpatients and 258 hospitalizations, respectively, per 100,000 inhabitants. Development of de novo resistance alone increases the total number of outpatients by about $6 \%$ and hospitalizations by about $21 \%$. If a resistant infection is introduced into the population after three weeks, the outcome dramatically deteriorates. Wide-spread use of $\mathrm{NI}$ treatment makes it highly likely that the resistant strain will spread if its fitness is high. This situation is further aggravated if a resistant virus is imported into a country in the early phase of an outbreak. As NI-resistant influenza infections with high fitness and pathogenicity have just been observed, the emergence of drug resistance in treated populations and the transmission of drug resistant strains is an important public health concern for seasonal and pandemic influenza.
\end{abstract}

\section{Findings}

Neuraminidase inhibitors (NI) play an important role in plans to mitigate future influenza pandemics [1]. Modeling studies suggested that a pandemic may be contained at the source, if treatment and prophylaxis are applied in an early phase of the epidemic. Large amounts of NI (mainly oseltamivir) have been stockpiled in many countries to prepare for pandemic influenza, and many national preparedness plans rely on this. However, recently doubts have been raised whether this strategy is realistic. Timeliness of the intervention due to difficulties in early recognition and logistic challenges are some of the points considered. The development of NI resistance is of further concern.

Influenza viruses undergo continuous genetic changes by means of mutation and recombination, promoting the emergence of drug resistant strains. Viral resistance may develop by modifications in the amino acid composition of the neuraminidase or in the affinity of haemagglutinin to the receptors of the cell surface [reviewed in [2]]. Prior to the $2007 / 8$ influenza season, NI resistant strains were 
found in patients after treatment with oseltamivir and in patients not exposed to oseltamivir. Resistance to NI occurred at a low level: less then $1 \%$ of immuno-competent patients were found to be infected with resistant virus [3]. The emergence of a resistant strain may not necessarily be dangerous, as the "fitness" of the resistant strain determines its transmissibility $[4,5]$. Most resistant strains lacked "fitness" and were unlikely to spread, but early surveillance data from the 2007/8 influenza season on the northern hemisphere suggest that an oseltamivir resistant influenza virus type $\mathrm{A}(\mathrm{H} 1 \mathrm{~N} 1)$ circulates in several European countries and in the US [6,7]. The proportion of resistant infections ranges between $4 \%$ and $67 \%$ (mean $20 \%$, approximately 1.700 tested isolates) and have been reported from 15 of 25 European countries under surveillance [8].

To obtain a better understanding of the consequences associated with the widespread use of NI as first-line option against a novel pandemic influenza strain, we extend the freely available simulation program InfluSim to simulate the emergence and spread of NI resistant strains $[9,10]$. We examine how the numbers of outpatients and hospitalizations change if resistance emerges de novo and is imported into a population in the early phase of an outbreak. We compare scenarios with and without the presence of drug resistance, using a basic reproduction number $R_{0}$ of 2.5 [11]. $R_{0}$ is the expected number of secondary infections per case in a completely susceptible population without interventions (it is calculated as the maximum eigenvalue of the next generation matrix) $[12,13]$. The fitness of the resistant infection, i.e. its capability to spread from person to person, is assumed to be the same as that of the drug sensitive one. Concordant to historical data and most pandemic plans [see $[13,14]]$, we assume that one third of all infected individuals remain asymptomatic, one third becomes moderately sick and one third becomes severely sick and seeks medical help. All cases who seek medical help ('outpatients') are offered antiviral treatment, and we assume that the NI stockpile is sufficiently large. General (unspecified) social distancing measures $[15,16]$ are simulated by reducing the number of contacts within the population by $10 \%$. Isolation additionally reduces the number of contacts of moderately sick cases by $10 \%$, of severe cases who stay at home by
$20 \%$, and of hospitalized cases by $30 \%$. On day zero, a single infection (which is either drug sensitive or drug resistant) is introduced into a fully susceptible population. On day 21, a second introduction follows (again drug sensitive or resistant). Drug resistance is assumed to develop additionally de novo during the course of the pandemic wave (we assume that $4.1 \%$ of children and teenagers and $0.32 \%$ of adults [cf. [17-19]] infected with the drug sensitive virus develop a resistant infection when taking antiviral drugs. Cases infected with the resistant virus do no longer respond to antiviral treatment. We report the incidence and total number of outpatients and hospitalizations during the course of the pandemic wave in a Swiss population of 100,000 inhabitants. The emergence and the initial spread of drug resistance are highly stochastic. Deterministic simulations as those presented here give average or mean courses of the resulting dynamics, but do not show the full stochastic range of results.

Without drug resistance, the simulated influenza epidemic causes 19,500 outpatients and 258 hospitalizations per 100,000 inhabitants. If only drug sensitive infections are imported, and drug resistance develops only de novo, the number of outpatients increases to $20,700(106 \%)$ and the number of hospitalizations increases to 312 (121\%; Table 1). If resistant infections do not only develop de novo, but are imported into the population 21 days after onset of the epidemic, the numbers rise to $22,700(116 \%)$ outpatients and to $420(163 \%)$ hospitalizations. If the resistant strain is imported before the drug sensitive one, numbers even rise to 25,100 (129\%) outpatients and 601 (233\%) hospitalizations, (Table 1). The latter values do not change if the resistant strain is imported a second time on day 21 .

If a resistant strain emerges only de novo, its prevalence may remain low, implying little epidemiological consequences (Figure 1a). Importation of resistance, however, increasingly replaces the drug sensitive strain because the latter is continuously eliminated by treatment. The dominance of the resistant strain depends on when its importation starts. E. g. if a drug resistant strain is imported 21 days after seeding the epidemic (with a sensitive strain), the prevalence curve for the resistant strain mimics in a delayed shape the prevalence of the sensitive strain (Fig-

Table I: Expected number of outpatients and hospitalizations in various scenarios with drug resistant infections

\begin{tabular}{cccc}
\hline | st infection imported on day 0 & 2nd infection imported on day 2| & Total number of outpatients & Total number of hospitalizations \\
\hline sensitive strain & sensitive strain & 20,700 & 313 \\
sensitive strain & resistant strain & 22,700 & 420 \\
resistant strain & sensitive strain & 25,100 & 601 \\
resistant strain & resistant strain & 25,100 & $60 \mid$
\end{tabular}

All patients who seek medical help ('outpatients') are offered antiviral treatment. The scenario without drug resistant infection leads to 19,500 outpatients and 258 hospitalizations, respectively. Population size 100,000; parameter values see Figure I and text. 

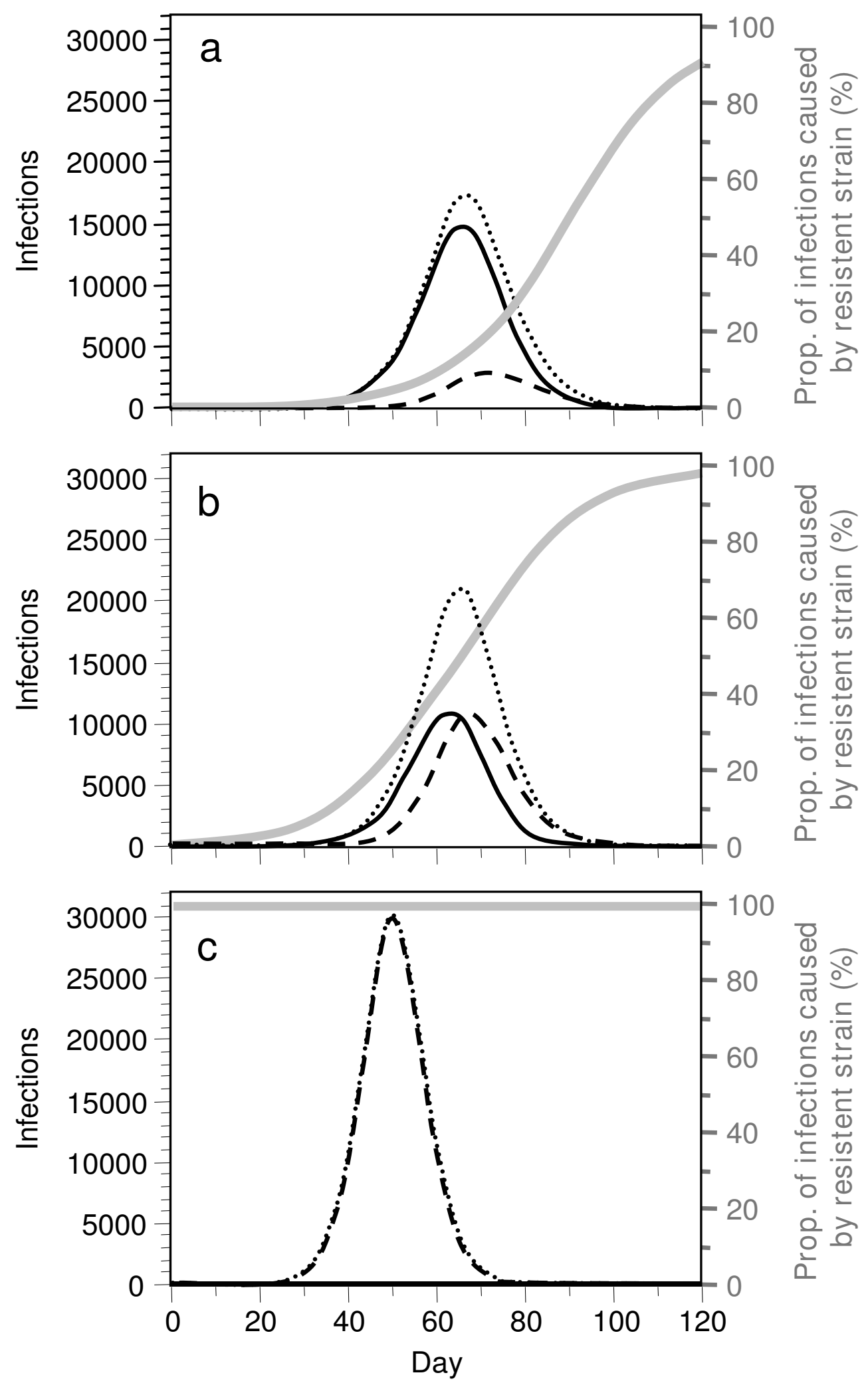

Figure I (see legend on next page) 
Figure I (see previous page)

Prevalence of infection with the drug sensitive virus (solid lines in black), the drug resistant one (dashed lines) and the sum of both (dotted lines). All cases who seek medical help ('outpatients') receive antiviral treatment. The grey curves indicate the fractions of resistant infections among all infections. In all 3 graphs, resistance develops de novo in $4.1 \%$ of children and $0.32 \%$ of adults who receive treatment. (a) Drug-sensitive infections are imported on day 0 and 21 ; (b) Drug sensitive infection is imported on day 0 , followed by a drug-resistant one on day 21 ; (c) Drug resistant infection is imported on day 0 , followed by a drug-sensitive one on day 2I. Further assumptions: (I) Swiss population of 100,000 individuals. (2) $R_{0}=2.5$ for the drug sensitive and the drug resistant virus. Both strains are assumed to have the same transmissibility. (3) One third of all infected individuals become severely sick and seek medical help. Antiviral treatment reduces their contagiousness by $80 \%$ and their duration of sickness by $25 \%$ if they are infected with the drug sensitive virus. (4) General social distancing reduces the number of contacts by $10 \%$ for all individuals; isolation additionally prevents $10 \%, 20 \%$ and $30 \%$ of contacts of moderately sick cases, severely sick cases at home, and hospitalized cases, respectively. For references about assumptions and parameter values see text.

ure $1 \mathrm{~b})$. If the time point for the importation of the resistant strain is shifted towards the initial phase of the epidemic, the resistant strain increasingly replaces the sensitive strain (Figure 1c). Early importation of resistant infection increases the number of treatment failures and thus, increases the overall number of infections emerging from the epidemic (Figure 1a-c). A sensitivity analysis which addresses the influence of the non-pharmaceutical interventions on these results is presented as an additional file (Additional file 1). Figure 2 illustrates the total numbers of (a) outpatients, (b) hospitalizations, and (c) deaths in dependence of a given time delay between the importation of the drug sensitive and the drug resistant infection (0-30 days).

Current mathematical models focus more on de novo drug resistance than on imported and spreading resistant infections [5,20,21]. Although de novo development of NI resistance may occur so late within a treated patient that the patient is unlikely to pass on the infection, widespread use of treatment makes it highly likely that resistant virus will circulate in the population if its relative fitness is high. We show in our simulations, that the development of de novo resistance on a low level and the subsequent spread of resistant virus results in a substantially increased number of hospitalizations, and subsequently in more ICU patients and deaths. Especially the shortcoming in the availability of intensive care beds has to be considered $[22,23]$. This situation is aggravated if an already resistant virus is imported into a population in the early phase of an epidemic. Up to now, only little attention has been paid to such scenarios. Observations in the early phase of the 2007/8 influenza season showed a marked increase of oseltamivir resistant influenza A virus (H1N1) in various European countries. The current oseltamivir resistant virus does not pose any risk to cause a pandemic as the $\mathrm{H} 1 \mathrm{~N} 1$ strain has been circulating in the population for many years without pandemic potential and leaving the population at least partially immune. The implications for avian influenza H5N1 remain uncertain.
Oseltamivir resistance due to the same mutation has been reported in three patients with $\mathrm{H} 5 \mathrm{~N} 1$ infection who were treated with oseltamivir. As $\mathrm{H} 5 \mathrm{~N} 1$ viruses have not yet shown the ability to spread efficiently from person to person there seems currently no potential for a similar increase. However, the appearance of a spreading NIresistant seasonal influenza strain is unexpected and of great concern. It highlights that even in the absence of widespread NI use for treatment or prophylaxis, oseltamivir resistant strains can emerge and spread in the population [6]. It also highlights the importance of our simulations for the elaboration of appropriate control and prevention strategies. We point out that the early introduction of a resistant influenza virus with pandemic potential may easily become an overwhelming public health problem. An increase of infections of $30 \%$ and a more than doubled total number of hospitalizations demonstrate this challenge. Non-pharmaceutical interventions considered by health decision makers and occupational medicine specialists in their pandemic preparedness plans may play a crucial role.

\section{List of abbreviations}

NI: Neuraminidase inhibitors; $R_{0}$ : basic reproduction number.

\section{Competing interests}

The authors declare that they have no competing interests.

\section{Authors' contributions}

SOB and ME conceived the research question of the study, analyzed the simulation results and drafted the manuscript. ME and MS formulated and programmed the model in Java and delivered the simulation results. HPD participated in the design of the study, performed the statistical analysis, produced the figure and helped to draft the manuscript. DK, MW and BV participated in its design and coordination and helped to draft the manuscript. All authors read and approved the final manuscript. 

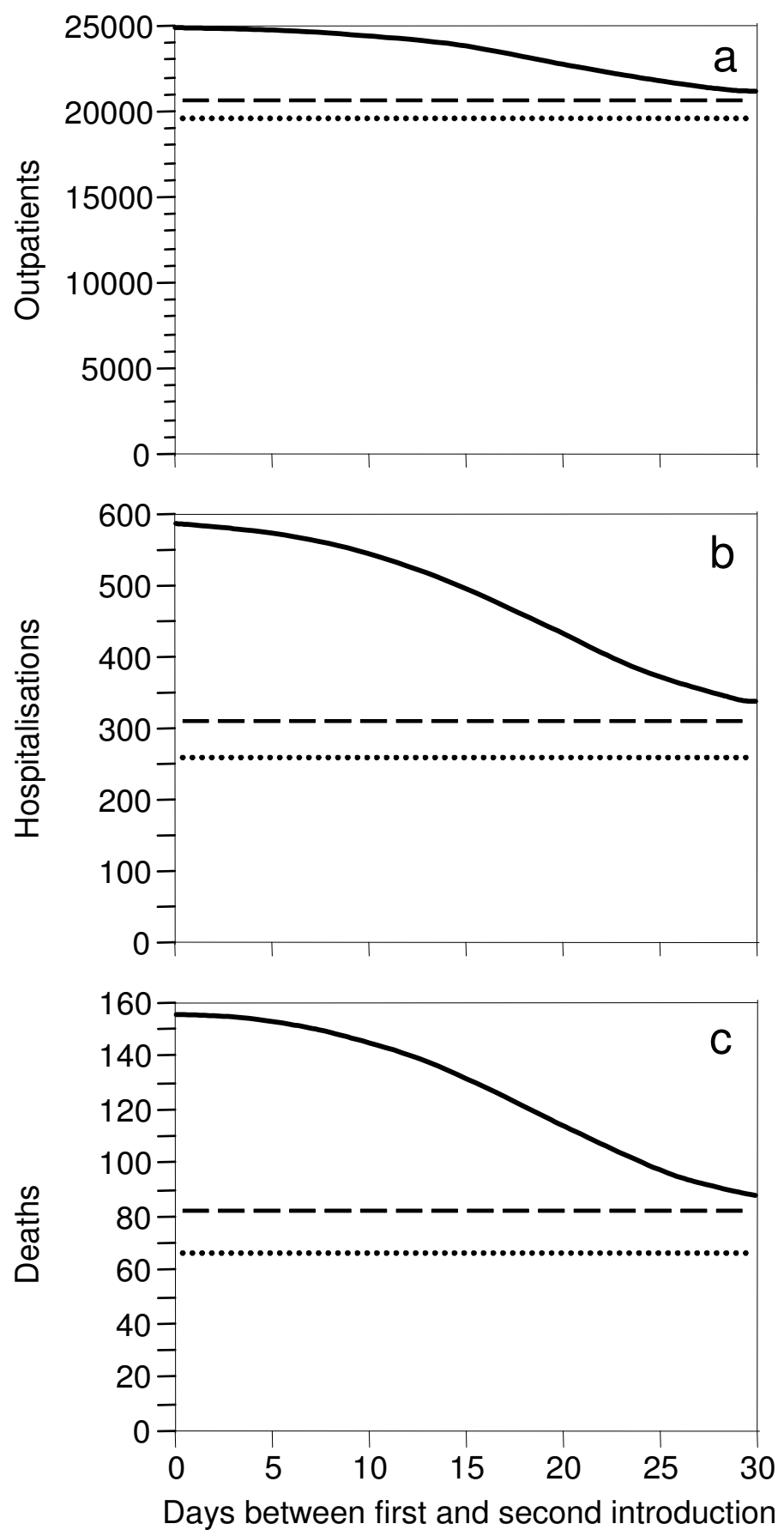

Figure 2

The solid curves show the expected total numbers of (a) outpatients, (b) hospitalizations, and (c) deaths, respectively, during a pandemic wave in a population of 100,000 inhabitants where on day 0 a drug-sensitive infection is imported, followed by a drug-resistant one after the time delay given on the horizontal axis. Without introduction of a resistant infection, 20,700 outpatients, 314 hospitalizations and 82 deaths are expected (dashed reference lines). If resistant infection is neither introduced de novo nor imported, 19,500 outpatients, 258 hospitalizations and 66 deaths are expected (dotted reference lines). Parameter values see Figure I and text. 


\section{Additional material}

\section{Additional File 1}

Sensitivity analysis on the influence of social distancing measures in the comparison of reintroduction of drug sensitive and drug resistant infection. The data provided represent the sensitivity analysis on the influence of social distancing measures on the number of outpatients and hospitalizations.

Click here for file

[http://www.biomedcentral.com/content/supplementary/1743422X-5-133-S1.doc]

\section{Acknowledgements}

This work has been partly supported by a project of the SFOPH (contract no. 06.001333/304.000I-108), the EU projects SARScontrol (FP6 STREP; contract no. 003824) (HPD) and INFTRANS (FP6 STREP; contract no. 5I37/5) (MS). We thank M. Mäusezahl and HC Matter for their support and for reviewing a previous version of the manuscript.

\section{References}

I. Strengthening pandemic-influenza preparedness and response, including application of the International Health Regulations (2005). Fifty-ninth World Health Assembly. WHO document A59/4 [http://www.who.int/gb/ebwha/pdf files/ WHA59/A59 4-en.pdf]

2. Updated review of influenza antiviral medicinal products for potential use during pandemic by the Committee for Medicinal Products for Human Use (CHMP) of the European Medicines Agency (EMEA) [http://www.emea.europa.eu/htms/ human/pandemicinfluenza/antivirals.htm]

3. Monto AS, McKimm-Breschkin JL, Macken C, Hampson AW, Hay A, Klimov A, Tashiro M, Webster RG, Aymard M, Hayden FG, Zambon $M$ : Detection of influenza viruses resistant to neuraminidase inhibitors in global surveillance during the first 3 years of their use. Antimicrob Agents Chemother 2006, 50:2395-2402.

4. Herlocher ML, Truscon R, Elias S, Yen HL, Roberts NA, Ohmit SE, Monto AS: Influenza viruses resistant to the antiviral drug oseltamivir: transmission studies in ferrets. J Infect Dis 2004, 190:1627-1630.

5. Stilianakis NI, Perelson AS, Hayden FG: Emergence of drug resistance during an influenza epidemic: insights from a mathematical model. J Infect Dis 1998, 177:863-873.

6. Interim ECDC Risk Assessment - Emergence of seasonal influenza viruses type $A / H I N I$ with oseltamivir resistance in some European Countries at the start of the 2007-8 influenza season [http://www.ecdc.europa.eu/pdf/080127 os.pdf]

7. Lackenby A, Hungnes O, Dudman SG, Meijer A, Paget WJ, Hay AJ, Zambon MC: Emergence of resistance to oseltamivir among influenza A(HINI) viruses in Europe. Euro Surveill 2008, I3(5):

8. Update $A(H I N I)$ viruses resistant to oseltamivir [http://
[ www.eiss.org $/ \mathrm{html} / / \mathrm{lb} \mathrm{h} / \mathrm{nl}$ resistance oseltamivir.html]

9. Ward P, Small I, Smith J, Suter P, Dutkowski R: Oseltamivir (Tamiflu) and its potential for use in the event of an influenza pandemic. J Antimicrob Chemother 2005, 55(SuppI I):i5-i2I.

10. Whitley RJ, Hayden FG, Reisinger KS, Young N, Dutkowski R, Ipe D, Mills RG, Ward P: Oral oseltamivir treatment of influenza in children. Pediatr Infect Dis J 200I, 20:127-I33.

II. Aoki FY, Boivin G, Roberts N: Influenza virus susceptibility and resistance to oseltamivir. Antivir Ther 2007, 12:603-6/6.

12. Dietz K: The estimation of the basic reproduction number for infectious diseases. Stat Methods Med Res 1993, 2:23-4I.

13. Eichner M, Schwehm M, Duerr HP, Brockmann SO: The influenza pandemic preparedness planning tool InfluSim. BMC Infect Dis 2007, 7:17.

14. Duerr HP, Brockmann SO, Piechotowski I, Schwehm M, Eichner M: Influenza pandemic intervention planning using InfluSim: pharmaceutical and non-pharmaceutical interventions. $B M C$ Infect Dis 2007, 7:76.
15. Jefferson T, Foxlee R, Del Mar C, Dooley L, Ferroni E, Hewak B, Prabhala $A$, Nair $S$, Rivetti $A$ : Interventions for the interruption or reduction of the spread of respiratory viruses. Cochrane Database Syst Rev 2007:CD006207.

16. Sande M van der, Teunis P, Sabel R: Professional and home-made face masks reduce exposure to respiratory infections among the general population. PLOS ONE 2008, 3:e26|8.

17. Chowell G, Nishiura H, Bettencourt LM: Comparative estimation of the reproduction number for pandemic influenza from daily case notification data. J R Soc Interface 2007, 4:I55-I66.

18. Ferguson NM, Cummings DA, Cauchemez S, Fraser C, Riley S, Meeyai A, lamsirithaworn S, Burke DS: Strategies for containing an emerging influenza pandemic in Southeast Asia. Nature 2005, 437:209-2।4.

19. Longini IM Jr, Halloran ME, Nizam A, Yang Y: Containing pandemic influenza with antiviral agents. $A m$ J Epidemiol 2004, 159:623-633.

20. Ferguson NM, Mallett S, Jackson H, Roberts N, Ward P: A population-dynamic model for evaluating the potential spread of drug-resistant influenza virus infections during communitybased use of antivirals. J Antimicrob Chemother 2003, 5 I:977-990.

21. Regoes RR, Bonhoeffer S: Emergence of drug-resistant influenza virus: population dynamical considerations. Science 2006, 3 | 2:389-39|.

22. Menon DK, Taylor BL, Ridley SA: Modelling the impact of an influenza pandemic on critical care services in England. Anaesthesia 2005, 60:952-954.

23. van Genugten ML, Heijnen ML: The expected number of hospitalisations and beds needed due to pandemic influenza on a regional level in the Netherlands. Virus Res 2004, 103:17-23.
Publish with Bio Med Central and every scientist can read your work free of charge

"BioMed Central will be the most significant development for disseminating the results of biomedical research in our lifetime."

Sir Paul Nurse, Cancer Research UK

Your research papers will be:

- available free of charge to the entire biomedical community

- peer reviewed and published immediately upon acceptance

- cited in PubMed and archived on PubMed Central

- yours - you keep the copyright
BioMedcentral 